\title{
Transition Metal Stabilized Arenium Cations: Protonation of Arenes Dihapto-Coordinated to $\square$-Basic Metal Fragments
}

Joseph M. Keane, Mahendra D. Chordia, Christopher J. Mocella, Michal Sabat, Carl O. Trindle, and W. Dean Harman

\section{Supplemental Materials}

General Methods. NMR spectra were obtained on a 300 or $500 \mathrm{MHz}$ Varian INOVA spectrometer. All chemical shifts are reported in ppm and are referenced to tetramethylsilane utilizing residual ${ }^{1} \mathrm{H}$ or ${ }^{13} \mathrm{C}$ signals of the deuterated solvents as an internal standard. Coupling constants $(J)$ are reported in hertz $(\mathrm{Hz})$. Carbon signals associated with triflate groups were not observed for any sample. Infrared spectra (IR) were recorded on a MIDAC Prospect Series (Model PRS) spectrometer as a glaze on a Horizontal Attenuated Total Reflectance (HATR) accessory (Pike Industries).

Electrochemical experiments were performed under a dinitrogen atmosphere using either a PAR model 362 potentiostat driven by a PAR model 175 universal programmer and Kipp and Zonen BD90 XY recorder, or a BAS Epsilon EC-2000 potentiostat. Cyclic voltametric $(\mathrm{CV})$ data was taken at $100 \mathrm{mV} / \mathrm{s}$ at ambient temperature in a standard threeelectrode cell from +1.7 to $-1.7 \mathrm{~V}$ with a glassy carbon working electrode, $N, N$ dimethylacetamide (DMA) solvent, and tetrabutylammonium hexaflurophosphate (TBAH) electrolyte $(\sim 0.5 \mathrm{M})$. All potentials are reported versus NHE (Normal Hydrogen Electrode) using cobaltocenium hexafluorophosphate $\left(\mathrm{E}_{1 / 2}=-0.78 \mathrm{~V}\right)$ or ferrocene $\left(\mathrm{E}_{1 / 2}=\right.$ $0.55 \mathrm{~V}$ ) as an internal standard. The peak-to-peak separation was less than $100 \mathrm{mV}$ for all reversible couples. Elemental analysis (EA) was obtained from Atlantic Microlabs, Inc. X-ray data was collected on a Bruker SMART APEX CCD diffractometer. Unless otherwise noted, all synthetic reactions and electrochemical experiments were performed under a dry nitrogen atmosphere. $\mathrm{CH}_{2} \mathrm{Cl}_{2}$, benzene, tetrahydrofuran (THF), and hexanes were purged with nitrogen and purified by passage through a column packed with activated alumina. ${ }^{1}$ Other solvents and liquid reagents were thoroughly purged with nitrogen prior to use. Deuterated solvents were used as received from Cambridge Isotopes. Diphenylammonium triflate was obtained by collecting the precipitate resulting from slow addition of triflic acid to a methylene chloride solution of diphenylamine. Compounds $1,{ }^{2} 2,{ }^{2} 4,{ }^{2} 13,{ }^{2} \mathrm{H}-13,{ }^{3} 14,{ }^{4} \mathrm{H}-14,{ }^{3}$ and $15^{5}$ have been reported previously. DFT calculations were performed on a Parallel Quantum Solutions (Fayetteville, AK) Linux system with INTEL processors.

$\left[\mathbf{T p R e}(\mathrm{CO})(\mathrm{BuIm})\left(\mathbf{3 , 4 , 5}-\square^{3}-(\mathbf{6 H}\right.\right.$-toluenium $\left.)\right](\mathrm{OTf})(\mathbf{2 H}-\mathbf{6})$ To TpRe$(\mathrm{CO})(\mathrm{BuIm})\left(\square^{2}-\right.$ toluene $)\left(6,0.011 \mathrm{~g}, 1.2 \times 10^{-5} \mathrm{~mol}\right)$ at $-40^{\circ} \mathrm{C}$ was added a solution of methanesulfonic acid $\left(0.010 \mathrm{~g}, 1.0 \times 10^{-4} \mathrm{~mol}\right)$ in $\mathrm{CDCl}_{3}(0.771 \mathrm{~g})$ at $-40{ }^{\circ} \mathrm{C}$. The resulting dark orange solution was observed at $-20^{\circ} \mathrm{C}$ by NMR spectroscopy. This compound was observed as a 1:1 mixture of isomers. ${ }^{1} \mathrm{H}$ NMR (acetone- $\left.d_{6},-20{ }^{\circ} \mathrm{C}, \square\right):(6 \mathbf{H}-6 \mathrm{~A}$, select resonances) $6.03(1 \mathrm{H}, \mathrm{d}, J=4.5, \mathrm{H} 2), 5.28$ (1H, bs, H5), 4.77 (1H, t, J=6.0, H4), 3.82 (1H, bs, H3), $3.20(1 \mathrm{H}, \mathrm{d}, J=25.0, \mathrm{H} 6), 3.12\left(1 \mathrm{H}\right.$, buried, H6), $2.23\left(3 \mathrm{H}, \mathrm{s}, \mathrm{CH}_{3}\right)$. (6H-6B, select resonances) $6.12(1 \mathrm{H}$, buried, $\mathrm{H} 2), 5.13(1 \mathrm{H}, \mathrm{bs}, \mathrm{H} 5), 4.72(1 \mathrm{H}, \mathrm{t}, J=6.0, \mathrm{H} 4), 4.52(1 \mathrm{H}$, bs, H3), $3.31(1 \mathrm{H}, \mathrm{d}, J=24.0, \mathrm{H} 6), 3.13\left(1 \mathrm{H}\right.$, buried, H6), $2.29\left(3 \mathrm{H}, \mathrm{s}, \mathrm{CH}_{3}\right)$. 
$\operatorname{TpRe}(\mathbf{C O})(\mathbf{B u I m})\left(\square^{2}\right.$-(o-xylene)) (7) To TpRe(CO)(BuIm) $\left(\square^{2}\right.$-benzene) (5, $0.211 \mathrm{~g}$, $\left.3.35 \times 10^{-4} \mathrm{~mol}\right)$ in a $125 \mathrm{~mL}$ Erlenmeyer flask charged with a stirbar was added $o$-xylene $(36.886 \mathrm{~g}, 0.348 \mathrm{~mol})$. The resulting yellow mixture was stirred at ambient temperature for 3 days, during which time it became a red solution. This solution was transferred to a $30 \mathrm{~mL}$ silica plug packed with $o$-xylene in a fritted Buchner funnel. The plug was eluted with $5 \% o$-xylene in ether, and a bright yellow band $(25 \mathrm{~mL})$ was collected. To this solution was added hexanes $(175 \mathrm{~mL})$, and the resulting solution was chilled by solvent evaporation to give $125 \mathrm{~mL}$ of a yellow slurry. A yellow precipitate was collected by filtration, rinsed with hexanes, and dried in vacuo to give a yellow solid $(0.081 \mathrm{~g}, 36.8 \%)$. This compound was observed as a 1.6:1 ratio of diastereomers with the major (7A) having the metal fragment bound between the xylene 4 and 5 positions, designated such that the methyl group at the 1 position is directed between the $\mathrm{CO}$ ligand and the imidazole ligand, and the minor (7B) having the metal fragment bound between the xylene 5 and 6 positions, designated such that the methyl group at the 1 position is directed toward the imidazole ligand. The presence of a small amount of a third isomer having the metal fragment bound between the xylene 5 and 6 positions, designated such that the methyl group at the 1 position is directed away from the imidazole ligand, could not be exhaustively ruled out. ${ }^{1} \mathrm{H}$ NMR (acetone- $d_{6},-20{ }^{\circ} \mathrm{C}$, $\square$ ): (Major Diastereomer (7A)) $8.13(1 \mathrm{H}, \mathrm{bs}, \mathrm{Tp} 3,5), 7.96(1 \mathrm{H}, \mathrm{d}, J=1.5$, Tp 3,5), $7.84(1 \mathrm{H}, \mathrm{bs}, \mathrm{Tp} 3,5), 7.78(1 \mathrm{H}$, bs, Tp 3,5), $7.64(1 \mathrm{H}$, bs, Im 2), $7.47(1 \mathrm{H}, \mathrm{bs}, \mathrm{Tp} 3,5), 7.21(1 \mathrm{H}, \mathrm{bs}, \operatorname{Im} 4,5), 7.01(1 \mathrm{H}, \mathrm{m}$, Tp 3,5), 6.95 (1H, bs, H3), 6.74 (1H, bs, Im 4,5), 6.54 (1H, bs, H6), 6.33 (1H, bs, Tp 4), 6.25 (1H, bs, Tp 4), 6.03 (1H, t, J=2.0, Tp 4), 4.58 (1H, very broad, BH), 4.15 (2H, m, $\left.\mathrm{NCH}_{2}\right), 3.89$ (1H, bs, H5), $3.29\left(1 \mathrm{H}\right.$, bs, H4), 2.35 (3H, s, 1-Pos $\left.\mathrm{CH}_{3}\right), 2.34$ (3H, s, 2-Pos $\left.\mathrm{CH}_{3}\right), 1.77\left(2 \mathrm{H}, \mathrm{bs}, \mathrm{NCH}_{2} \mathrm{CH}_{2}\right), 1.26\left(2 \mathrm{H}, \mathrm{m}, \mathrm{CH}_{2} \mathrm{CH}_{3}\right), 0.92\left(3 \mathrm{H}, \mathrm{m}, \mathrm{CH}_{2} \mathrm{CH}_{3}\right)$. (Minor Diastereomer (7B), select resonances) $6.90(1 \mathrm{H}, \mathrm{bs}, \mathrm{H} 4), 5.94(1 \mathrm{H}, \mathrm{d}, J=8.5, \mathrm{H} 3), 4.05$ $(1 \mathrm{H}, \mathrm{m}, \mathrm{H} 6), 3.35(1 \mathrm{H}, \mathrm{m}, \mathrm{H} 5), 2.23\left(3 \mathrm{H}, \mathrm{s}, 2-\mathrm{Pos} \mathrm{CH}_{3}\right), 1.40\left(3 \mathrm{H}, \mathrm{bs}, 1-\mathrm{Pos} \mathrm{CH}_{3}\right) .{ }^{13} \mathrm{C}$ NMR (acetone- $d_{6},-20^{\circ} \mathrm{C}$, $\square$ ): (Major Diastereomer (7A)) 196.7 or $196.4(\mathrm{CO}$, other is minor), 143.7 (Tp 3,5), 142.7 (Tp 3,5), 140.2 (Im 2), 139.6 (Tp 3,5), 136.2 (Tp 3,5 and C3), 135.7 (Tp 3,5 and C6), $134.9(\operatorname{Tp} 3,5), 131.5(\operatorname{Im} 4,5), 120.79(\operatorname{Im} 4,5), 106.5(\operatorname{Tp} 4)$, 106.2 (Tp 4), 106.1 (Tp 4), 66.4 (C5), $64.2(\mathrm{C} 4), 47.5\left(\mathrm{NCH}_{2}\right), 33.6\left(\mathrm{CH}_{2} \mathrm{CH}_{2}\right), 19.9$ $\left(\mathrm{CH}_{2} \mathrm{CH}_{3}, 1-\mathrm{Pos} \mathrm{CH}_{3}, 2-\mathrm{Pos} \mathrm{CH}_{3}\right), 13.7\left(\mathrm{CH}_{2} \underline{\mathrm{CH}}_{3}\right),(\mathrm{C} 1$ and $\mathrm{C} 2$ buried or indiscernible from minor resonances). (Minor Diastereomer (7B), select resonances) 196.7 or 196.4 (CO, other is major), 134.9 (C4), 123.0 (C3), 73.4 (C6), 65.5 (C5), 19.7 (1-Pos $\mathrm{CH}_{3}$ ), $18.3\left(2-\mathrm{Pos}_{\mathrm{CH}_{3}}\right)$. IR $\square_{\mathrm{cO}}=1790 \mathrm{~cm}^{-1}$ (vs), $\square_{\mathrm{BH}}=2475 \mathrm{~cm}^{-1}(\mathrm{w}) . \mathrm{CV}: \mathrm{E}_{\mathrm{p}, \mathrm{a}}=-0.260 \mathrm{~V}$. EA: Calculated for $\mathrm{C}_{25} \mathrm{H}_{32} \mathrm{BN}_{8} \mathrm{ORe}$ : C, 45.66; H, 4.90; N, 17.04. Found C, 45.40; H, $5.04 ; \mathrm{N}, 17.25$.

$\operatorname{TpRe}(\mathbf{C O})(\mathbf{B u I m})\left(\mathbf{5 , 6}-\square^{2}\right.$-(m-xylene)) (8) To TpRe(CO)(BuIm) $\left(\square^{2}\right.$-benzene) $(\mathbf{5}, 0.218$ $\left.\mathrm{g}, 3.47 \times 10^{-4} \mathrm{~mol}\right)$ in a $125 \mathrm{~mL}$ Erlenmeyer flask charged with a stirbar was added $\mathrm{m}$ xylene $(49.683 \mathrm{~g}, 0.469 \mathrm{~mol})$. The resulting yellow mixture was stirred at ambient temperature for 3 days, during which time it became an orange solution. This solution was transferred to a $30 \mathrm{~mL}$ silica plug packed with $m$-xylene in a fritted Buchner funnel. The plug was eluted with $10 \% \mathrm{~m}$-xylene in hexanes $(10 \mathrm{~mL})$, followed by ether. A yellow band $(50 \mathrm{~mL})$ was collected. To this solution was added hexanes $(150 \mathrm{~mL})$, and the resulting solution was chilled by solvent evaporation to give $150 \mathrm{~mL}$ of a yellow 
slurry. A yellow precipitate was collected by filtration, rinsed with hexanes, and dried in vacuo to give a yellow solid $(0.068 \mathrm{~g})$. This material was estimated to be $\sim 60 \%$ pure by electrochemistry and ${ }^{1} \mathrm{H}$ NMR integrations, with the remainder consisting primarily of $\left[\mathrm{TpRe}(\mathrm{CO})\left(\mu-3-\square^{1}-4,5-\square^{2}-\mathrm{BuIm}\right)\right]_{2}(\mathbf{1 D})$ and $\mathrm{TpRe}(\mathrm{CO})(\mathrm{BuIm})\left(\mathrm{N}_{2}\right)$. This estimate suggests a yield of $\sim 18 \%$. This compound was observed as a facial isomer having the methyl group at the one position directed toward the imidazole ligand. The presence of small amounts of a second isomer having the methyl group at the 1 position directed away from the imidazole ligand could not be exhaustively ruled out. ${ }^{1} \mathrm{H}$ NMR (acetone$d_{6},-20{ }^{\circ} \mathrm{C}$, ฤ): (Select resonances) $6.66(1 \mathrm{H}, \mathrm{d}, J=4.5, \mathrm{H} 4), 5.69(1 \mathrm{H}, \mathrm{s}, \mathrm{H} 2), 4.15(2 \mathrm{H}$, m, $\left.\mathrm{NCH}_{2}\right), 3.92(1 \mathrm{H}, \mathrm{d}, J=8.0, \mathrm{H} 6), 3.34(1 \mathrm{H}, \mathrm{dd}, J=8.0,4.5, \mathrm{H} 5), 2.29$ (3H, s, 3-pos $\left.\mathrm{CH}_{3}\right), 1.76\left(2 \mathrm{H}, \mathrm{m}, \mathrm{NCH}_{2} \underline{\mathrm{C}}_{2}\right), 1.44\left(3 \mathrm{H}, \mathrm{s}, 1-\operatorname{pos} \mathrm{CH}_{3}\right), 1.28\left(2 \mathrm{H}, \mathrm{m}, \mathrm{C}_{2} \mathrm{CH}_{3}\right), 0.90$ $\left(3 \mathrm{H}, \mathrm{m}, \mathrm{CH}_{2} \underline{\mathrm{C}}_{3}\right)$. IR $\square_{\mathrm{CO}}=1791 \mathrm{~cm}^{-1}(\mathrm{vs}), \square_{\mathrm{BH}}=2468 \mathrm{~cm}^{-1}(\mathrm{w}) . \mathrm{CV}: \mathrm{E}_{\mathrm{p}, \mathrm{a}}=-0.190 \mathrm{~V}$.

$\left[\mathrm{TpRe}(\mathrm{CO})(\mathrm{BuIm})\left(5,6-\Gamma^{2}-(2 H-3-\right.\right.$ methylanisolium $\left.\left.)\right)\right](\mathrm{OTf})(2 \mathrm{H}-10)$ and $\left[\operatorname{TpRe}(\mathrm{CO})(\mathrm{BuIm})\left(\mathbf{5 , 6}-\square^{2}-(\mathbf{H} \boldsymbol{H}-3-\right.\right.$ methylanisolium $\left.\left.)\right)\right](\mathrm{OTf})(\mathbf{4 H}-10)$ These complexes were observed under a variety of conditions. In a prototypical experiment, to $\mathrm{TpRe}(\mathrm{CO})(\mathrm{BuIm})\left(5,6-\square^{2}-(3\right.$-methylanisole $\left.)\right)\left(\mathbf{1 0}, 0.010 \mathrm{~g}, 1.5 \times 10^{-5} \mathrm{~mol}\right)$ and diphenylammonium triflate $\left(0.006 \mathrm{~g}, 2 \times 10^{-5} \mathrm{~mol}\right)$ was added acetone- $d_{6}(0.5 \mathrm{~g})$, and the resulting dark red solution was observed by NMR spectroscopy. This compound was observed as a 12:3:3:1 mixture of isomers consisting of a $4 \mathrm{H}$ isomer having the $\mathrm{OCH}_{3}$ group directed toward the imidazole ligand $(\mathbf{4 H - 1 0 A})$, a $2 \mathrm{H}$ isomer having the $\mathrm{OCH}_{\mathrm{s}}$ group directed toward the imidazole ligand $(\mathbf{2 H - 1 0 A})$, a $4 \mathrm{H}$ isomer having the $\mathrm{OCH}_{3}$ group directed away from the imidazole ligand $(\mathbf{4 H}-\mathbf{1 0 B})$, and a $2 H$ isomer having the $\mathrm{OCH}_{3}$ group directed away from the imidazole ligand $(\mathbf{2 H}-\mathbf{1 0 B})$, respectively. ${ }^{1} \mathrm{H}$ NMR (acetone- $d_{6}$, ambient temperature, $\square$ ): (Major Isomer $(\mathbf{4 H - 1 0 A})$ ) $8.09(1 \mathrm{H}, \mathrm{d}, J=2.5$, Tp 3,5), $8.07(1 \mathrm{H}, \mathrm{d}, J=2.5$, Tp 3,5), $7.97(1 \mathrm{H}, \mathrm{dd}, J=2.5,0.5 \mathrm{Tp} 3,5), 7.90(1 \mathrm{H}, \mathrm{d}, J=2.0$, Tp 3,5), $7.81(1 \mathrm{H}, \mathrm{dd}, J=2.5,0.5, \mathrm{Tp} 3,5), 7.40(1 \mathrm{H}, \mathrm{d}, J=2.0, \mathrm{Tp} 3,5), 7.38(1 \mathrm{H}, \mathrm{t}, J=2.0$, Im), 6.69 (1H, bs, Im), $6.53(1 \mathrm{H}, \mathrm{t}, J=2.5$, Tp 4), $6.51(1 \mathrm{H}, \mathrm{bs}, \mathrm{H} 2), 6.36(1 \mathrm{H}, \mathrm{t}, J=2.5$, Tp 4), 6.15 (1H, t, $J=2.5$, Tp 4), $4.33(1 \mathrm{H}, \mathrm{d}, J=7.5, \mathrm{H6}), 4.20\left(2 \mathrm{H}, \mathrm{t}, J=7.5, \mathrm{NCH}_{2}\right), 3.98$ $(1 \mathrm{H}, \mathrm{td}, J=7.5,1.5, \mathrm{H} 5), 3.67(1 \mathrm{H}, \mathrm{d}, J=25.0, \mathrm{H} 4), 3.63\left(3 \mathrm{H}, \mathrm{s}, \mathrm{OCH}_{3}\right), 3.62(1 \mathrm{H}, \mathrm{d}$, $J=18.5, \mathrm{H} 4), 2.40\left(3 \mathrm{H}, \mathrm{s}, 3-\operatorname{pos} \mathrm{CH}_{3}\right), 1.79\left(2 \mathrm{H}, \mathrm{m}, \mathrm{NCH}_{2} \mathrm{C}_{2}\right), 1.26\left(2 \mathrm{H}, \mathrm{m}, \mathrm{C}_{2} \mathrm{CH}_{3}\right)$, $0.89\left(3 \mathrm{H}, \mathrm{m}, \mathrm{CH}_{2} \mathrm{CH}_{3}\right)(1 \mathrm{x} \mathrm{Im}$ buried or indiscernible from minor resonances; $\mathrm{BH}$ not observed). (2H-10A, Select Resonances) 4.85 (1H, d, J=7.0, H6), 4.10 (1H, m, H5), 3.77 $\left(3 \mathrm{H}, \mathrm{s}, \mathrm{OCH}_{3}\right), 2.35\left(3 \mathrm{H}, \mathrm{s}, 3-\right.$ pos $\left.\mathrm{CH}_{3}\right)$. (4H-10B, Select Resonances) 4.84 (1H, t, J=7.5, H5), $3.41(1 \mathrm{H}, \mathrm{dt}, J=7.5,1.5, \mathrm{H} 6), 3.15\left(3 \mathrm{H}, \mathrm{s}, \mathrm{OCH}_{3}\right), 2.32\left(3 \mathrm{H}, \mathrm{s}, 3-\mathrm{pos} \mathrm{CH}_{3}\right)$. (2H10B, Select Resonances) $5.01(1 \mathrm{H}, \mathrm{m}, \mathrm{H} 5), 3.19\left(3 \mathrm{H}, \mathrm{s}, \mathrm{OCH}_{3}\right) .{ }^{13} \mathrm{C} \mathrm{NMR}$ (acetone- $d_{6}$, $15{ }^{\circ} \mathrm{C}$, $\square$ ): (Major Isomer (4H-10A)) 196.0 (CO), 192.4 (C1), 167.6 (C3), 144.1 (Tp 3,5), $143.8(\operatorname{Tp~3,5),~} 142.4(\operatorname{Tp} 3,5), 138.1(\mathrm{Tp} 3,5), 137.7(\mathrm{Tp} 3,5), 136.0(\mathrm{Tp} 3,5), 131.3$ (Im), 125.2 (Im), 122.6 (Im), 119.5 (C2), 107.8 (Tp 4), 107.6 (Tp 4), 107.4 (Tp 4), 61.4 (C5), $58.7\left(\mathrm{OCH}_{3}\right), 55.6(\mathrm{C} 6), 48.1\left(\mathrm{NCH}_{2}\right), 39.6(\mathrm{C} 4), 33.3\left(\mathrm{NCH}_{2} \underline{\mathrm{CH}_{2}}\right), 24.1$ (3-pos $\left.\mathrm{CH}_{3}\right), 19.9\left(\mathrm{CH}_{2} \mathrm{CH}_{3}\right), 13.6\left(\mathrm{CH}_{2} \underline{\mathrm{CH}}_{3}\right)$.

[TpRe(CO)(BuIm)(5,6- $\square^{2}-(\mathbf{2 H}-4$-methylanisolium) $\left.)\right](\mathrm{OTf})(\mathbf{2 H}-\mathbf{1 1})$ This complex was observed under a variety of conditions. In a prototypical experiment, to $\mathrm{TpRe}(\mathrm{CO})(\mathrm{BuIm})\left(5,6-\square^{2}-(4-m e t h y l a n i s o l e)\right)\left(\mathbf{1 1}, 0.011 \mathrm{~g}, 1.6 \times 10^{-5} \mathrm{~mol}\right)$ and diphenylammonium triflate $\left(0.006 \mathrm{~g}, 2 \times 10^{-5} \mathrm{~mol}\right)$ was added acetone- $d_{6}(0.3 \mathrm{~g})$, and the 
resulting dark red solution was observed by NMR spectroscopy. This compound was observed as a 3:1 mixture of diastereomers, the major $(\mathbf{2 H - 1 1 B})$ having the $\mathrm{OCH}_{3}$ group directed away from the imidazole ligand, and the minor $(\mathbf{2 H - 1 1 A})$ having the $\mathrm{OCH}_{3}$ group directed toward the imidazole ligand. ${ }^{1} \mathrm{H}$ NMR (acetone- $d_{6}$, ambient temperature, П): (Major Diastereomer (2H-11B)) $8.38(1 \mathrm{H}, \mathrm{d}, J=2.0$, Tp 3,5), 8.12 (1H, d, J=2.0, Tp 3,5), $8.06(1 \mathrm{H}, \mathrm{d}, J=1.5$, Tp 3,5), $8.01(1 \mathrm{H}, \mathrm{t}, J=2.5$, Im), 7.92 (1H, t, $J=3.0, \mathrm{Im}), 7.80$ $(1 \mathrm{H}, \mathrm{d}, J=2.0, \operatorname{Tp} 3,5), 7.51(1 \mathrm{H}, \mathrm{d}, J=2.5, \mathrm{Im}), 7.50(1 \mathrm{H}, \mathrm{d}, J=2.5 \mathrm{Tp} 3,5), 7.38(1 \mathrm{H}, \mathrm{bs}$, Tp 3,5), 7.11, $6.62(1 \mathrm{H}, \mathrm{t}, J=2.5$, Tp 4), $6.42(1 \mathrm{H}, \mathrm{t}, J=2.5$, Tp 4), 6.17 (1H, t, $J=2.5$, Tp 4), 5.00 (1H, d, J=5.5, H5), $4.83(1 \mathrm{H}, \mathrm{m}, \mathrm{H} 3), 4.20\left(2 \mathrm{H}, \mathrm{m}, \mathrm{NCH}_{2}\right), 3.90(1 \mathrm{H}, \mathrm{dd}, J=6.5$, 1.5, H6), $3.38(1 \mathrm{H}, \mathrm{d}, J=27.5, \mathrm{H} 2), 3.19\left(3 \mathrm{H}, \mathrm{s}, \mathrm{OCH}_{3}\right), 3.13(1 \mathrm{H}, \mathrm{d}, J=27.5, \mathrm{H} 2), 1.78$ $\left(2 \mathrm{H}, \mathrm{m}, \mathrm{NCH}_{2} \mathrm{CH}_{2}\right), 1.27\left(2 \mathrm{H}, \mathrm{m}, \mathrm{CH}_{2} \mathrm{CH}_{3}\right), 1.25\left(3 \mathrm{H}, \mathrm{s}, 4-\operatorname{pos} \mathrm{CH}_{3}\right), 0.88(3 \mathrm{H}, \mathrm{m}$, $\mathrm{CH}_{2} \mathrm{CH}_{3}$ ), (BH not observed). (Minor Diastereomer (2H-11A), Select Resonances) 4.96 (1H, bs, H3), 4.79 (1H, d, J=7.0, H6), $3.82\left(3 \mathrm{H}, \mathrm{s}, \mathrm{OCH}_{3}\right), 3.81$ (1H, buried, H5), 3.53 $(1 \mathrm{H}, \mathrm{d}, J=27.5, \mathrm{H} 2), 3.33\left(1 \mathrm{H}\right.$, buried, H2), $1.36\left(3 \mathrm{H}, \mathrm{s}, 4-\operatorname{pos} \mathrm{CH}_{3}\right) .{ }^{13} \mathrm{C}$ NMR (acetone$d_{6},-20{ }^{\circ} \mathrm{C}$, \): (Major Diastereomer (2H-11B)) 196.2 (C1), 194.5 (CO), 146.6 (Tp 3,5), 144.2 (Tp 3,5), $141.0(\mathrm{Tp} 3,5), 139.6(\mathrm{Tp} 3,5), 137.8$ (Tp 3,5), 136.7 (Tp 3,5), 122.4 (Im), 111.1 (C4), 108.7 (Tp 4), 108.2 (Tp 4), 107.4 (Tp 4), 70.8 (C5), 62.2 (C6), 61.0 (C3), $58.2\left(\mathrm{OCH}_{3}\right), 48.1\left(\mathrm{NCH}_{2}\right), 34.0(\mathrm{C} 2), 33.4\left(\mathrm{NCH}_{2} \mathrm{CH}_{2}\right), 22.6\left(4-p o s \mathrm{CH}_{3}\right), 19.9$ $\left(\mathrm{CH}_{2} \mathrm{CH}_{3}\right), 13.6\left(\mathrm{CH}_{2} \underline{\mathrm{CH}}_{3}\right),(2 \mathrm{x}$ Im indiscernible from minor resonances).

$\left[\mathbf{T p R e}(\mathbf{C O})(\mathbf{B u I m})\left(\square^{3}-\mathbf{C}_{\mathbf{6}} \mathbf{H}_{\mathbf{9}}\right)\right](\mathbf{O T f})(\mathbf{1 7})$ To a mixture of $\mathrm{TpRe}(\mathrm{CO})(\mathrm{BuIm})\left(\square^{2}-\right.$ benzene) $\left(5,0.192 \mathrm{~g}, 3.05 \times 10^{-4} \mathrm{~mol}\right)$ and diphenylammonium triflate $(0.119 \mathrm{~g}, 3.73 \mathrm{x}$ $\left.10^{-4} \mathrm{~mol}\right)$ was added benzene $(15.979 \mathrm{~g})$, and the resulting yellow mixture was stirred for 25 minutes, during which time it became a red solution. This solution was added to hexanes $(200 \mathrm{~mL})$, and the resulting precipitate was collected by filtration, rinsed with hexanes, and dried in vacuo to give a tan solid $(.212 \mathrm{~g})$. The purity of this material is thought to be low $(<50 \%)$ based on stoichiometric considerations of its proposed mechanism of formation. Assignments are made considering metal fragment to be bound at the 4,5 , and 6 ring positions. ${ }^{1} \mathrm{H}$ NMR (acetone- $d_{6}$, ambient temperature, $\square$ ): 8.49 $(1 \mathrm{H}, \mathrm{d}, J=1.5$, Tp 3,5), $8.18(1 \mathrm{H}, \mathrm{d}, J=2.1$, Tp 3,5), 8.01 (1H, d, J=2.1, Tp 3,5), $7.93(1 \mathrm{H}$, d, $J=2.1$, Tp 3,5), 7.91 (1H, bs, Im), $7.84(1 \mathrm{H}, \mathrm{bs}, \mathrm{Tp} 3,5), 7.69$ (1H, d, $J=1.8 \mathrm{Tp} 3,5)$, $7.31(1 \mathrm{H}, \mathrm{bs}, \mathrm{Im}), 6.72(1 \mathrm{H}, \mathrm{bs}, \mathrm{Im}), 6.67(1 \mathrm{H}, \mathrm{bs}, \mathrm{Tp} 4), 6.38(1 \mathrm{H}, \mathrm{bs}, \mathrm{Tp} 4), 6.30(1 \mathrm{H}$, bs, Tp 4), $4.97(1 \mathrm{H}, \mathrm{t}, J=6.3, \mathrm{H} 5), 4.85(1 \mathrm{H}, \mathrm{m}, \mathrm{H} 6), 4.22(1 \mathrm{H}, \mathrm{m}, \mathrm{H} 4), 4.12(2 \mathrm{H}, \mathrm{t}, J=7.2$, $\left.\mathrm{NCH}_{2}\right), 3.32(1 \mathrm{H}, \mathrm{m}, \mathrm{H} 1), 3.26(1 \mathrm{H}, \mathrm{m}, \mathrm{H} 1), 2.28(1 \mathrm{H}, \mathrm{m}, \mathrm{H} 1), 2.23(1 \mathrm{H}, \mathrm{m}, \mathrm{H} 3), 1.71$ $\left(2 \mathrm{H}, \mathrm{m}, \mathrm{NCH}_{2} \mathrm{CH}_{2}\right), 1.50(1 \mathrm{H}, \mathrm{m}, \mathrm{H} 2), 1.26(1 \mathrm{H}, \mathrm{m}, \mathrm{H} 2), 1.17\left(2 \mathrm{H}, \mathrm{m}, \mathrm{C}_{2} \mathrm{CH}_{3}\right), 0.85$ (3H, t, $\left.J=7.5, \mathrm{CH}_{2} \underline{\mathrm{C}}_{3}\right)$, (BH not observed).

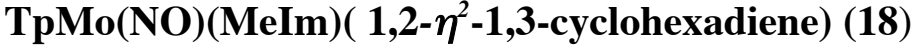

To a solution of TpMo(NO)(MeIm)Br $(0.929 \mathrm{~g}, 1.85 \mathrm{mmol})$ in $100 \mathrm{~mL}$ of THF in a 250 $\mathrm{mL}$ round-bottom flask, $3.11 \mathrm{~g}(38.8 \mathrm{mmol})$ of cyclohexadiene and $31.2 \mathrm{~g}$ of $1.05 \%$ $\mathrm{Na} / \mathrm{Hg}$ amalgam were added. The mixture quickly turned from green to purple, and was allowed to stir for $24 \mathrm{~h}$. The THF solution was decanted away from the amalgam, and the solvent was evaporated. The residue was dissolved in a minimal amount of THF and loaded onto a 2" silica gel plug in a $60 \mathrm{~mL}$ fritted funnel. The beige product band was collected by elution with a 1:3 (v/v) THF:diethyl ether solution. The eluent was reduced to $10 \mathrm{~mL}$ at which time pentane $(100 \mathrm{~mL})$ was added to precipitate a beige solid that was 
subsequently collected by filtration. The solid was dried under reduced pressure. Typical yield: $65-70 \%$.

$\mathrm{CV} \mathrm{E}_{\mathrm{p}, \mathrm{a}}=+0.155 \mathrm{~V}$. IR: $\square(\mathrm{BH})=2479 \mathrm{~cm}^{-1} \square(\mathrm{NO})=1557 \mathrm{~cm}^{-1} .{ }^{1} \mathrm{H}$ NMR (acetonitrile$\left.d_{3}\right)\left(22^{\circ} \mathrm{C}\right) \square 7.96,7.86,7.85,7.82,7.80,7.73,7.72,7.71,7.60,7.47,7.26,7.17$ (1H each, each a d, Tp 3,5), 6.33, 6.13, 6.11 (1H each, 1:1:1, each a t, Tp 4), 6.27 (3H, m, Tp 4), 7.66, 7.52, 7.34, 7.05, 7.01, 6.63 (1H each, each a br s, Im), $3.65(3 \mathrm{H}, \mathrm{s}, \mathrm{NMe}) .2 .47(1 \mathrm{H}$, br dt, J = $9 \mathrm{~Hz}, \mathrm{C} 1), 1.93(1 \mathrm{H}, \mathrm{br} \mathrm{dt}, \mathrm{J}=9 \mathrm{~Hz}, \mathrm{C} 1$ '), $2.31(1 \mathrm{H}, \mathrm{dd}, \mathrm{J}=9 \mathrm{~Hz}, 5 \mathrm{~Hz}, \mathrm{C} 2)$, $2.50(1 \mathrm{H}, \mathrm{dd}, \mathrm{J}=9 \mathrm{~Hz}, 5 \mathrm{~Hz}, \mathrm{C} 2$ '), $6.33(1 \mathrm{H}, \mathrm{ddd}, \mathrm{J}=10 \mathrm{~Hz}, 6 \mathrm{~Hz}, 3 \mathrm{~Hz}, \mathrm{C} 3), 5.98(1 \mathrm{H}$, ddd, J = 10 Hz, 6 Hz, 3 Hz, C3'), 5.17 (1H, ddd overlapping, J=19 Hz, 4 Hz, 2 Hz, C4), 5.15 (1H, ddd overlapping, J=19 Hz, 4 Hz, 2 Hz, C4'), 2.44 (2H, m, C5/C5'), 1.78 (2H, m, C5/C5'), 2.68 (1H, tdd, J = 14 Hz, 7 Hz, 4 Hz, C6), 1.82 (1H, ddt, J = 14 Hz, 7 Hz, C6), 2.61 (1H, tdd, J = 14 Hz, 7 Hz, 4 Hz, C6'), 1.99 (1H, ddt, J = 14 Hz, 7 Hz, 4 Hz, C6'). ${ }^{13} \mathrm{C}$ NMR $(300 \mathrm{MHz})\left(\mathrm{d}_{3}\right.$-acetonitrile) $\left(22^{\circ} \mathrm{C}\right) \square 145.0,143.0,142.5,142.1,142.0$, 141.9, 139.1, 138.6, 137.6, 137.2, 136.8, 135.9, 135.8, 133.1, 132.9 (12 Tp 3, 5, 2 Im, 2 unbound $\mathrm{CH}), 129.8,129.3,122.9,122.4$ (4 Im), 118.6, 118.1 (2 unbound $\mathrm{CH}), 106.7$, 106.6, 106.4, 106.5 (4 Tp 4), 71.9, 66.5, 63.9, 60.3 (4 bound $\mathrm{CH}), 34.8,34.7\left(2 \mathrm{Im} \mathrm{CH}_{3}\right)$, 27.0, 26.9, 24.3, $23.8\left(4 \mathrm{CH}_{2}\right)$. Anal. Calcd. For: $\mathrm{C}_{19} \mathrm{H}_{24} \mathrm{BMoN}_{9} \mathrm{O}: \mathrm{C}, 45.53 ; \mathrm{H}, 4.83 ; \mathrm{N}$, 25.15. Found: C, 45.39; H, 4.81; N, 25.09.

\section{TpMo(NO)(MeIm)(1,2,3- $\square^{\beta}-4 H$-cyclohexadienyl) triflate (19)}

To an acetonitrile $(10 \mathrm{~mL})$ solution of $18(0.385 \mathrm{~g}, 0.768 \mathrm{mmol})$ in a $125 \mathrm{~mL}$ flask, $1.24 \mathrm{~g}$ $(0.855 \mathrm{mmol})$ of $10.3 \%$ (wt/wt) of triflic acid in methanol was added dropwise with stirring. The solution instantly changed from a light yellow to an orange color. The solution was stirred for 5 minutes, and the solvent was removed in vacuo. The resulting residue was dissolved in $5 \mathrm{~mL}$ of $\mathrm{THF}$, and pentane $(75 \mathrm{~mL})$ was added to afford a yellow-orange precipitate that was collected and dried under reduced pressure. Typical yield: $80 \%$.

CV: $\mathrm{E}_{\mathrm{p}, \mathrm{c}}=-0.781 \mathrm{~V}, \mathrm{E}_{\mathrm{p}, \mathrm{a}}=+0.027 \mathrm{~V} . \mathrm{IR}: \square(\mathrm{BH})=2500 \mathrm{~cm}^{-1} \square(\mathrm{NO})=1657 \mathrm{~cm}^{-1} \cdot{ }^{1} \mathrm{H}$ NMR (acetonitrile- $\left.d_{3} ; 22{ }^{\circ} \mathrm{C}\right) \square 8.01,7.99,7.91,7.90,7.74,7.49,7.35(1 \mathrm{H}$ each, each a d, $6 \mathrm{Tp} 3,5,1 \mathrm{Im}), 7.10,6.56$ (1H each, each a t, Im), 6.50, 6.40, 6.18 (1H each, each a $\mathrm{t}, \mathrm{Tp}$ 4), $5.93(1 \mathrm{H}, \mathrm{dd}, \mathrm{J}=6 \mathrm{~Hz}, 2.5 \mathrm{~Hz}, \mathrm{C} 3), 5.69(1 \mathrm{H}, \mathrm{dd}, \mathrm{J}=7.5 \mathrm{~Hz}, 1.5 \mathrm{~Hz}, \mathrm{C} 1), 4.97(1 \mathrm{H}, \mathrm{t}$, $\mathrm{J}=7 \mathrm{~Hz}, \mathrm{C} 2), 1.47$ (1H, m, C5), 1.73 (1H, m, C5'), 3.24 (1H, m, C4), 3.10 (1H, m, C6), 2.79 (1H, m, C4'), 2.72 (1H, m, C6'). ${ }^{13} \mathrm{C}$ NMR (300 MHz) (d $\mathrm{d}_{2}$-methylene chloride) $\left(22^{\circ} \mathrm{C}\right) \square 146.1,142.9,142.3,138.6,138.4,138.2,135.7$ (6 Tp 3,5, $\left.1 \mathrm{Im}\right), 128.3,123.5$ (2 Im), 107.9, 107. 2, 106.9 (3 Tp 4), 125.6 (C3), 109.7 (C1), 103.5 (C2), $35.4\left(\mathrm{Im} \mathrm{CH}_{3}\right)$, 26.5 (C4), 26.4 (C6), 19.7 (C5). Anal. Calcd. For: $\mathrm{C}_{20} \mathrm{H}_{25} \mathrm{BF}_{3} \mathrm{MoN}_{9} \mathrm{O}_{4} \mathrm{~S} \mathrm{C}, 36.88 ; \mathrm{H}, 3.87$; N, 19.36. Found: C, 36.80; H, 3.88; N, 19.41.

$\operatorname{TpRe}(\mathbf{C O})(\mathbf{B u I m})\left(\mathbf{N}_{2}\right)$ This complex was observed as a trace product in the synthesis of $\mathrm{TpRe}(\mathrm{CO})(\mathrm{BuIm})\left(5,6-\Gamma^{2}-(\mathrm{m}-\mathrm{xylene})\right)(8)$ and other procedures. ${ }^{1} \mathrm{H}$ NMR (acetone- $d_{6}$, ambient temperature, $\square): 7.88(1 \mathrm{H}, \mathrm{dd}, J=2.5,1.0, \mathrm{Tp} 3,5), 7.84(1 \mathrm{H}, \mathrm{dd}, J=2.0,0.5$, Tp 3,5), $7.77(1 \mathrm{H}, \mathrm{dd}, J=2.5,1.0, \mathrm{Tp} 3,5), 7.68(1 \mathrm{H}, \mathrm{d}, J=2.0$, Tp 3,5), 7.49 (1H, t, $J=1.5$, Im 2), $7.34(1 \mathrm{H}, \mathrm{d}, J=2.0, \mathrm{Tp} 3,5), 7.32(1 \mathrm{H}, \mathrm{d}, J=2.0$, Tp 3,5) 7.19 (1H, t, $J=1.5, \operatorname{Im} 4,5)$, $6.79(1 \mathrm{H}, \mathrm{t}, J=1.5, \mathrm{Im} 4,5), 6.28(1 \mathrm{H}, \mathrm{t}, J=2.5$, Tp 4), $6.20(1 \mathrm{H}, \mathrm{t}, J=2.5$, Tp 4), $6.19(1 \mathrm{H}$, $\mathrm{t}, J=2.5, \mathrm{Tp} 4), 4.12\left(2 \mathrm{H}, \mathrm{t}, J=7.0, \mathrm{NCH}_{2}\right), 1.77\left(2 \mathrm{H}, \mathrm{m}, \mathrm{NCH}_{2} \mathrm{CH}_{2}\right), 1.28(2 \mathrm{H}, \mathrm{m}$,

$\left.\mathrm{C}_{2} \mathrm{CH}_{3}\right), 0.91\left(3 \mathrm{H}, \mathrm{t}, J=8.0, \mathrm{CH}_{3}\right) .{ }^{13} \mathrm{C} \mathrm{NMR}$ (acetone- $d_{6}$, ambient temperature, $\left.\square\right): 145.2$ (Tp 3,5 or Im), 144.1 (Tp 3,5 or Im), 141.6 (Tp 3,5 or Im), 141.2 (Tp 3,5 or Im), 136.0 
(Tp 3,5 or Im), 135.9 (Tp 3,5 or Im), 135.6 (Tp 3,5 or Im), 133.3 (Im), 121.1 (Im), 106.9 (Tp 4), 106.7 (2 x Tp 4), $47.9\left(\mathrm{NCH}_{2}\right), 33.6\left(\mathrm{NCH}_{2} \mathrm{CH}_{2}\right), 20.1\left(\mathrm{CH}_{2} \mathrm{CH}_{3}\right), 13.7\left(\mathrm{CH}_{3}\right),(\mathrm{CO}$ not observed). IR: $\square_{\mathrm{CO}}=1811 \mathrm{~cm}^{-1}(\mathrm{vs}), \square_{\mathrm{NN}}=1995 \mathrm{~cm}^{-1}(\mathrm{vs}), \square_{\mathrm{BH}}=2481 \mathrm{~cm}^{-1}(\mathrm{w}) . \mathrm{CV}$ : $\mathrm{E}_{\mathrm{p}, \mathrm{a}}=0.18 \mathrm{~V}$.

(1) Pangborn, A. B.; Giardello, M. A.; Grubbs, R. H.; Rosen, R. K.; Timmers, F. J. Organometallics 1996, 15, 1518.

(2) Meiere, S. H.; Brooks, B. C.; Gunnoe, T. B.; Carrig, E. H.; Sabat, M.; Harman, W. D. Organometallics 2001, 20, 3661.

(3) Valahovic, M. T.; Gunnoe, T. B.; Sabat, M.; Harman, W. D. J. Am. Chem. Soc. 2002, $124,3309$.

(4) Gunnoe, T. B.; Sabat, M.; Harman, W. D. Organometallics 2000, 19, 728.

(5) Meiere, S. H.; Keane, J. M.; Gunnoe, T. B.; Sabat, M.; Harman, W. D. J. Am. Chem. Soc. 2003, 125, 2024. 